\title{
Pilot study: Improving patient outcomes with healing touch
}

\author{
Kimberly Hendricks* and Kelley F Wallace \\ Fresenius Baton Rouge Home Program, 4848 Mancuso Lane, Suite B, Baton Rouge, Louisiana 70809, USA
}

\begin{abstract}
Healing Touch therapies use a practitioner's intentional placement of hands to influence energy fields of the patient to promote self-healing. In the changing climate of healthcare where patient experience increasingly drives reimbursements, it is vital to find unique and meaningful ways to ensure positive patient experiences beyond traditional medical therapies. To that end, our peritoneal dialysis clinic staff adopted Jean Watson's theoretical framework by which patients are treated as whole, unique persons with a "multidimensional system of energy, with a consciousness that can be affected by another to promote well-being,". With a rapidly growing urban clinic of more than 100 patients, our nurses identified the need to provide more ways to improve patient care.

Staff were educated on ways to set positive intentions for patient contacts and incorporate patient narratives into contact. Optional daily prayer was initiated, and patients were invited to attend a new support group for peritoneal dialysis patients and their families. In addition, a preliminary pilot provided healing touch treatments to interested patients. This pilot, described below, found improvement in 4 out of 5 measured categories. Fatigue and pain decreased by $46 \%$ and $68 \%$, respectively. Stress/anxiety fell by $48 \%$, while depression saw a large drop of $84 \%$. Nausea was unchanged.
\end{abstract}

\section{Introduction}

The United States Renal Data System reported in 2014 that ESRD beneficiaries, although fewer than $1 \%$ of the Medicare population, were responsible for over $\$ 32.8$ billion in spending - roughly $7.2 \%$ of Medicare fee-for-service spending [1,2]. This comes at a time when MedPAC projects hospitals should expect an average of $9 \%$ decrease in margins on Medicare business [3]. With the passage of the Patient Protection and Affordable Care Act of 2010, value-based purchasing now includes Hospital Consumer Assessment of Healthcare Providers and Systems surveys (HCAHPS) quality scores among others used to calculate payments. Up to $30 \%$ of the weight for these scores are based on the Patient Experience of Care [4].

In a chronic peritoneal dialysis (PD) patient, medical management may be sufficient to control fluid volume status, azotemia, and electrolyte imbalances sufficiently to prolong patients' lives. However, ensuring positive patient experiences and quality of life when faced with a chronic illness is more complex. To that end, our clinic initiated a holistic care approach, based on Jean Watson's Transpersonal Caring Model, to combat issues of fatigue, pain, stress and anxiety, and depression which may be more difficult to manage through traditional medical channels. Though this was a multi-prong approach, the most significant change made was the offering of healing touch therapy which recognizes the body as a complex energy system that can be improved through "use of intention and the placement of hands in specific sequences either above or on the body," [5]. A 10-session pilot study was conducted on the results of this new therapy offering.

\section{Methods}

We enlisted the services of a Level 5 Healing Touch (HT) practitioner to provide treatments to patients on a PRN basis, with six patients receiving sessions over the course of the pilot. Previously, HT practitioners have found this therapy promotes pain relief, reduced anxiety and improves relaxation [6]. Our practitioner broadened the scope to also include fatigue, depression and nausea which are common complaints among the chronic PD population.

Each HT session was approximately 30 to 45 minutes, using focused meditative intentions and a series of hand positions and movements on and around the patient to create a deep relaxation of mind, body and spirit. Treatments were performed in a designated space with a therapeutic milieu to promote relaxation and focus. Calming music was incorporated, along with dimmed lighting and a comfortable treatment table. In addition to pre- and post-vital signs, participants were surveyed directly before and after their treatments using a simple Likert scale to quantify changes to the target categories of fatigue, stress/ anxiety, pain, depression and nausea (Figure 1). It should be noted that this study is limited to qualitative, patient-reported data. Further investigation was not conducted on the effects of HT on physiological changes to the participants.

\section{Results}

Patient reports found little to no effect on nausea, and thus we will no longer pursue this area for improvement using healing touch. The largest improvement was found in an $84 \%$ reduction in depression, followed by $68 \%$ reduction in pain. Finally, stress/anxiety were reduced by $48 \%$ and fatigue by $46 \%$ (Figure 2 ).

Correspondence to: Kimberly Hendricks, Fresenius Baton Rouge Home Program, 4848 Mancuso Lane, Suite B, Baton Rouge, Louisiana 70809, USA, E-mail: kimberly.hendricks@fmc-na.com

Key words: healing touch, holistic, Jean Watson

Received: February 04, 2018; Accepted: February 25, 2018; Published: February 28,2018 


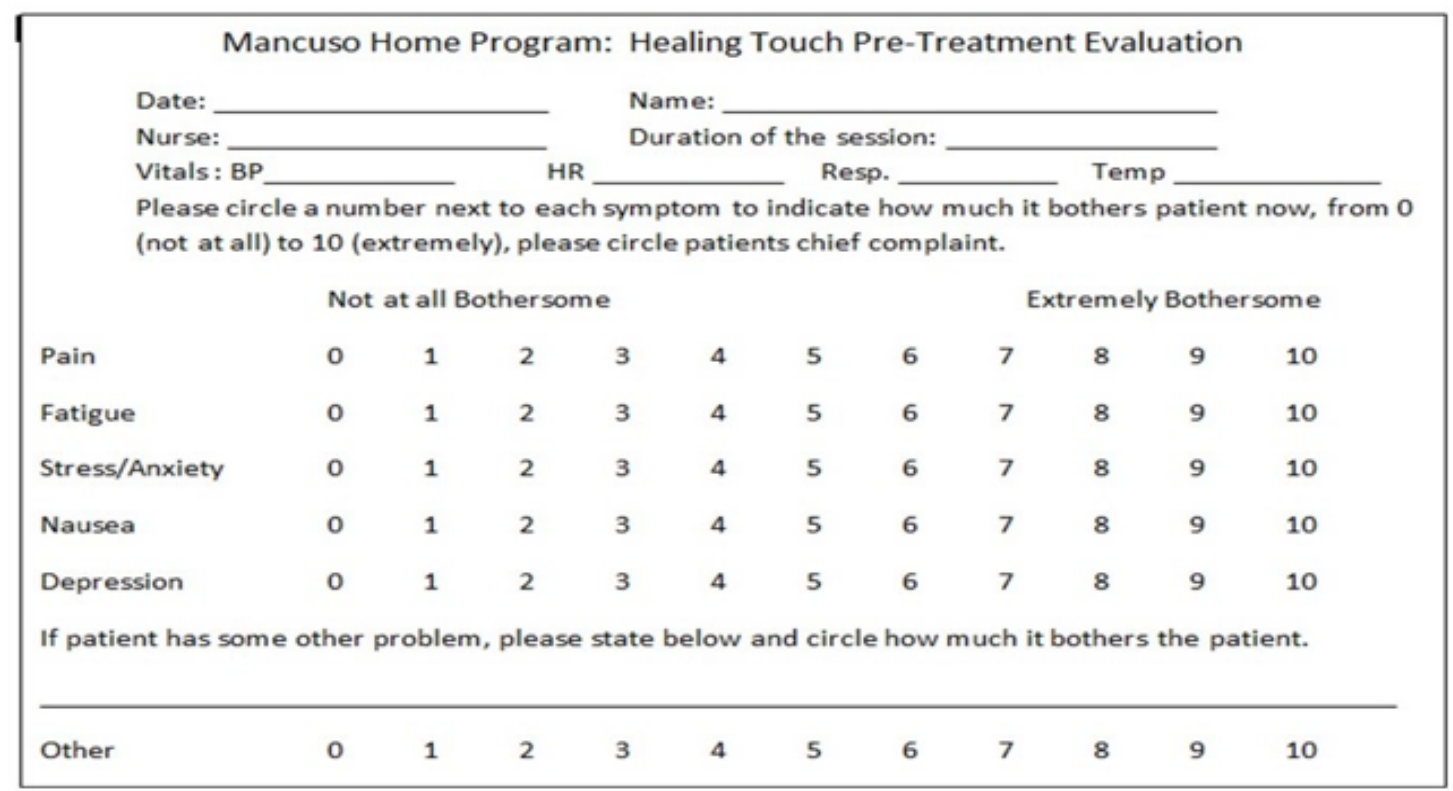

Figure 1. Pre-Treatment assessment tool, Adapted from Greenwich Hospital Integrative Medicine Program-Yale New Haven Health Systems Pilot study 2006.

\begin{tabular}{|l|c|c|c|c|}
\hline & $\begin{array}{c}\text { Pre } \\
\text { Assessment }\end{array}$ & $\begin{array}{c}\text { Post } \\
\text { Assessment }\end{array}$ & $\begin{array}{c}\text { Percent } \\
\text { Improvement }\end{array}$ & $\begin{array}{c}\text { p-Value } \\
\text { t-test }\end{array}$ \\
\hline Fatigue & 5.86 & 3.14 & $46 \%$ & 0.0028 \\
\hline Pain & 7.40 & 2.40 & $68 \%$ & 0.015 \\
\hline Stress / & & & & \\
Anxiety & 7.80 & 4.00 & $48 \%$ & 0.0058 \\
\hline & & & & \\
Depression & 6.25 & 1.00 & $84 \%$ & 0.0018 \\
\hline
\end{tabular}

Figure 2. Patient-reported improvement with statistical significance; $n=10$.

\section{Discussion}

The above data, though garnered from a small pilot study, presents promising uses for healing touch therapy in the chronic peritoneal dialysis population. Developing regular protocol for our patients suffering from pain, stress/anxiety, fatigue and depression could potentially provide significant benefits to an at-risk population. Although this study is the first noted within the peritoneal dialysis population, other studies have been shown to improve patient well-being using healing touch within other populations. Wardell and Weymouth provided a review of other disciplines previously incorporating healing touch, and found a variety of applications for healing touch from surgical recovery and pain relief to mood improvement and reduction in adverse outcomes [5]. Additionally, a completed literature review reported similar qualitative improvements in cancer patients with treatment-related symptoms such as nausea, fatigue and pain [7].

These findings warrant deeper investigation to determine whether these changes can improve patient outcomes on a broader level, and if patient experience can influence metrics such as hospitalizations, Kidney Disease Quality of Life Indicators (KDQOL), and more. Plans for growth of this therapy include training of additional nursing staff in healing touch therapies, expansion of therapeutic space designated for patient treatment rooms, and more robust education of patients, family members and staff to understand how healing touch therapies augment and supplement the traditional medical therapies currently in practice.

\section{References}

1. Swengros D, Herbst AM, Friesen MA, Mangione L, Anderson JG (2014) Promoting Caring-Healing Relationships. Holist Nurs Pract 28: 370-375. [Crossref]

2. Innovation Center Home 2017.

3. Hospital Medicare margins are projected to drop -- big time 2016.

4. https://www.cms.gov/Medicare/Quality-Initiatives-Patient-Assessment- Instruments/ HospitalQualityInits/HospitalHCAHPS.html

5. Wardell DW, Weymouth KF (2004) Review of Studies of Healing Touch. J Nurs Scholarsh 36: 147-154. [Crossref]

6. Umbreit A (1997) Therapeutic touch: Energy-based healing. Creat Nurs 3: 6-8. [Crossref]

7. Gonella S, Garrino L, Dimonte V (2014) Biofield Therapies and Cancer-Related Symptoms: A Review. Clin J Oncol Nurs 18: 568-576. [Crossref]

Copyright: (C2018 Hendricks K. This is an open-access article distributed under the terms of the Creative Commons Attribution License, which permits unrestricted use, distribution, and reproduction in any medium, provided the original author and source are credited. 